\title{
Higher specialist training in diabetes and endocrinology in the UK: interpreting the YDEF National Training Survey 2014
}

\author{
PAUL V CARROLL
}

\begin{abstract}
Background
A key remit of the YDEF is to improve the quality of training and trainee experience for those undertaking higher specialist training in D\&E in the UK. The YDEF performed a national survey of trainees' perceptions of higher specialist training issues in $2014 .{ }^{1}$ Ninety-four (representing $20 \%$ ) of the approximately 460 NTN holders in D\&E in the UK completed a questionnaire exploring specific domains related to their experience of and satisfaction with their training. Completion of the annual GMC survey is mandatory for doctors in postgraduate training posts. ${ }^{2}$ Although there was considerable overlap of the domains examined by the two surveys, the YDEF survey provides more detailed information in key areas. The survey report concludes that there are significant but solvable problems with the delivery and experience of training for $\mathrm{D} \& \mathrm{E}$ trainees.
\end{abstract}

\section{Survey findings}

The overwhelming majority of D\&E specialist trainees in the UK undertake dual training alongside GIM leading to attainment of CCT in both GIM and D\&E. The YDEF survey highlighted concern amongst trainees that an imbalance continues between GIM and specialty-specific D\&E training. The report indicates that on average $68 \%$ of training time is spent within GIM and $32 \%$ dedicated to D\&E specifically. The authors commented that D\&E trainees spend significantly more time involved in GIM than other medical specialty trainees who achieve dual accreditation (e.g. gastroenterology and respiratory medicine), although no data were cited to support this. It is the general perception amongst trainees, consultants, and the SAC that D\&E doctors contribute substantially more to GIM service delivery than other specialties. There are several reasons for this, including expansion of trainee numbers to address the pressure on rotas caused by the implementation of the European Working Time Directive; this applied particularly to smaller district general hospitals. The shift of diabetic patients in particular from hospital outpatients to the community poses a further potential threat to

Guy's \& St Thomas' NHS Foundation Trust, London, UK

Address for correspondence: Dr Paul V Carroll Guy's \& St Thomas' NHS Foundation Trust, London, UK Tel: +44 (0)20 71887188

E-mail: paul.carroll@gstt.nhs.uk

Br J Diabetes Vasc Dis 2014:14:85-86

http://dx.doi.org/10.15277/bjdvd.2014.033

\author{
Abbreviations and acronyms \\ CCT \\ $D \& E$ \\ GIM \\ GMC \\ NTN \\ SAC \\ TPD \\ YDEF \\ certificate of completion of training \\ diabetes and endocrinology \\ general internal medicine \\ General Medical Council \\ National Training Number \\ specialist advisory committee \\ training programme director \\ Young Diabetologists' \& Endocrinologists' Forum
}

training opportunities in D\&E. The lack of a diagnostic or therapeutic 'procedure' undertaken by D\&E specialists means they, along with geriatricians, are regarded as better able to respond to pressures on the general medical inpatient crisis than their interventionist peers. The current 5 year higher specialty training programme is expected to include GIM activities for a minimum of 3 years, but the curriculum is 'competency' rather than 'time commitment' based, allowing other specialty trainees to attain the same CCT but serving less GIM time in training.

\section{Addressing the challenges}

Those involved in the organisation and delivery of D\&E higher specialist training programmes recognise that balance between GIM and D\&E training matters enormously. This is particularly the case for recruitment to the specialty, an area which is of real concern in D\&E. As a discipline becomes less attractive, posts become vacant, which can become occupied with less able, but NTN holding trainees. Maintaining the quality of the future D\&E consultant workforce is dependent on continually attracting the best trainees to the specialty. The attractiveness of training programmes is heavily influenced by GIM/D\&E balance. It is recognised that the 2010 D\&E curriculum needs re-consideration to reflect the changes in the organisation, delivery and skill-set in current and future clinical services. This is particularly the case with development in sub-specialisation, multidisciplinary meetings, obesity medicine and community diabetes services.

The YDEF survey highlighted that trainees in D\&E would welcome protected time from GIM to allow trainees to concentrate on obtaining specialty specific competences, clinical knowledge and non-clinical skills. Other specialty training times have generally achieved this concept of protected time within training programmes. The development of a D\&E curriculum that contained generic training competencies, perhaps alongside GIM activities, but then mandated specific D\&E training without other commit- 
ments might redress some of the balance between GIM and D\&E. The next version of the D\&E curriculum may therefore provide a real opportunity to consider helping trainees achieve sub-specialty skills, making themselves distinctive, and provide D\&E specific time within the 5 year programme. In the meantime, examples do exist throughout the UK where D\&E programmes contain time (almost exclusively in teaching hospitals) protected for D\&E specific training. The balance between teaching hospital and district general hospital rotations varies across the Deaneries/Lead Providers in the UK, again an influence on the attractiveness of training programmes. The Royal College of Physicians' ambition to maintain general medicine skills and training for all specialties provides simultaneous opportunities and threats for the ambition of protected time for training in D\&E. Solving the issue of balance in training for dual-accrediting D\&E trainees is not easy, but momentum is gathering for the creation of 'protected' specialty training time.

Other areas of concern for trainees identified in the survey related to clear training plans, access to study leave, educational supervision, and overall experience. The summary GMC survey, with a greater number of respondents, for D\&E (2014) reported no overall outliers in the key domains which included areas highlighted as meriting improvement in the YDEF survey. ${ }^{2}$ Despite differences in these findings, it is recognised that improvements could be made in the areas of creating training plans, career guidance, educational supervision and facilitating personal development for trainees in D\&E. It is interesting to see how few of the trainees report being involved in service development or improvement projects. The prevalence of diabetes in the hospital in-patient setting, safety and prescribing issues, new medications, integrated team working and the rigorous detail involved in clinical endocrinology all provide excellent opportunities for trainees to participate in governance, audit and quality improvement programmes. The reported low level of current involvement may be explained by perceived lack of opportunity, time issues related to GIM commitment or by lack of enthusiasm by the trainees themselves. The opportunities either exist or can be created and there is an onus on both the 'systems' and the trainees themselves to create, secure and deliver improvement projects. This is particularly the case in integrated and community diabetes care delivery, an increasing component of the activity of existing and newly appointed consultants. To date few trainees have had real experience in the design and delivery of community diabetes services, yet many will be involved in this clinical activity in their consultant role.

\section{Implications for the speciality}

The need to provide attractive, effective and competitive higher specialist training schemes for D\&E trainees in the UK is well recognised. The D\&E SAC discusses and seeks to improve the quality of training experience regularly, learning from best practice and informed by surveys such as this one from the YDEF. ${ }^{3}$ Lead providers, Deaneries and TPDs continually seek to refine and improve training programmes, recognising the changing skills required by future consultants. TPDs interact both formally and informally, sharing experiences and societies (e.g. Society for Endocrinology) facilitate meetings to discuss attracting and developing trainees to D\&E. Workforce planning and training programmes are under more scrutiny than ever before. Changes to the commissioning of postgraduate medical education in England and the emergence of Lead Providers delivering training programmes provide opportunities for changes to existing rotations and structures. As the report from the YDEF states this is a good time for trainees themselves to take the initiative and strive to make their training better. ${ }^{4,5}$

\section{References}

1. Grant P, Chakera A, Cheer K, et al. YDEF training survey 2014. Br J Diabetes Vasc Dis 2014;14:120-23.

http://dx.doi.org/10.15277/bjdvd.2014.025

2. GMC Training Survey 2014

http://www.gmc-uk.org/NTS_2014_KFR_A4.pdf_56706809.pdf

3. Royal College of Physicians. Future hospital: caring for medical patients. September 2013. https://www.rcplondon.ac.uk/sites/default/files/futurehospital-commission-report.pdf (accessed August 2014).

4. Price HC, George J, Wilmot EG, et al. Taking training into your own hands. Clin Med 2010;10:349-51.

http://dx.doi.org/10.7861/clinmedicine.10-4-349

5. Ahluwalia R, Atkin M, Gallen I, et al. Diabetes and endocrinology specialist registrars teaching programmes in the United Kingdom--time to set some quality standards? Results from the Association of British Clinical Diabetologists--Young Diabetologists Forum survey. Diabet Med 2012;29:553-4. http://dx.doi.org/10.1111/j.1464-5491.2011.03501.x 